\title{
An Iterative Particle Filter Approach for Respiratory Motion Estimation in Nuclear Medicine Imaging
}

\author{
Ashrani Aizzuddin Abd. Rahni ${ }^{* a}$, Kevin Wells ${ }^{a}$, Emma Lewis ${ }^{a}$, Matthew Guy ${ }^{b}$, Budhaditya \\ Goswami $^{\text {a }}$ \\ ${ }^{\mathrm{a} C e n t r e}$ for Vision, Speech and Signal Processing, University of Surrey, Guildford, Surrey, GU2 \\ $7 \mathrm{XH}, \mathrm{UK}$ \\ ${ }^{b}$ Medical Physics Department, Southampton University Hospital Trust, Southampton, SO16 6YD, \\ UK
}

\begin{abstract}
The continual improvement in spatial resolution of Nuclear Medicine (NM) scanners has made accurate compensation of patient motion increasingly important. A major source of corrupting motion in NM acquisition is due to respiration. Therefore a particle filter (PF) approach has been proposed as a powerful method for motion correction in NM. The probabilistic view of the system in the $\mathrm{PF}$ is seen as an advantage that considers the complexity and uncertainties in estimating respiratory motion. Previous tests using XCAT has shown the possibility of estimating unseen organ configuration using training data that only consist of a single respiratory cycle. This paper augments application specific adaptation methods that have been implemented for better PF estimates with an iterative model update step. Results show that errors are further reduced to an extent up to a small number of iterations and such improvements will be advantageous for the PF to cope with more realistic and complex applications.
\end{abstract}

Keywords: Particle Filter, Iterative Model Update, Respiratory Motion Correction, Nuclear Medicine Imaging

\section{INTRODUCTION}

Nuclear Medicine (NM) is currently recognized as the most sensitive approach for functional imaging of the human body. As such it is an important tool in oncology, especially in the early detection of cancerous lesions. Although there has been significant improvement in system spatial resolution and sensitivity, leading to reduced acquisition time on current NM scanners, scan times are still much longer than the period of a single breath-hold used in X-ray CT (i.e. typically 5-15 minutes for PET and 15-30 minutes for SPECT ${ }^{1}$ ). Together with the improvement in spatial image resolution, these considerations make patient motion an increasingly important issue to be addressed.

Respiratory motion dominates motion artefacts when imaging the torso. This paper continues the development of a motion correction framework that has been proposed in Wells et al., $2009^{2}$. In this framework, respiratory motion correction is proposed to be applied continuously, enabling the use of all potential data, for example, list mode data in NM before image reconstruction. This has been deemed most appropriate considering the complex nature of respiratory motion $^{2,3}$. A probabilistic view of the problem has also been incorporated into the proposed framework to acknowledge the inherent uncertainties and this forms the basis of the particle filter (PF) approach. In this approach, internal organ deformation is inferred from a stereo camera capture of the anterior portion of the external surface of the torso and from previous estimates of organ deformation. This inference is based on a state transition model, which relates the configuration of organs, $\mathbf{X}_{\mathrm{k}}$ at a discrete time index $k$ with those at $k-1$ and a measurement model, which correlates the state $\mathbf{X}_{\mathrm{k}}$ to the external observation $\mathbf{Z}_{\mathrm{k}}$. This paper follows the development of the framework presented in Abd. Rahni et $a l ., 2010^{4}$ with an additional step to update the models used in the PF.

In a clinical application, the transition model will be estimated from training data which consists of a low dose dynamic CT scan of a patient ${ }^{5}$. A simultaneous stereo camera capture of the external torso surface ${ }^{6}$ provides additional training data to estimate the measurement model and facilitate motion correction during the NM phase of data acquisition. During the NM acquisition, the stereo camera will continue to provide observations of the external torso surface for the PF to

*a.abdrahni@surrey.ac.uk

Medical Imaging 2011: Image Processing, edited by Benoit M. Dawant, David R. Haynor,

Proc. of SPIE Vol. 7962, 79624C · (C) 2011 SPIE · CCC code: 1605-7422/11/\$18 - doi: 10.1117/12.878086

Proc. of SPIE Vol. 7962 79624C-1 
estimate any internal organ deformation. Any deformation can thus be corrected so that all the NM counts can be summed to enable motion compensated reconstruction of the complete NM dataset (optimising the available signal-tonoise ratio). In this paper all training and test data have been generated using the XCAT digital phantom ${ }^{7}$ to simulate the clinical application. This framework is thus patient specific while accommodating intra-patient variation in respiratory motion.

In Section 2 that follows, the implementation of the PF is presented, including the additional step beyond the previous implementation in Abd. Rahni et al., 2010 $0^{4}$. Section 3 presents the datasets simulated from the XCAT digital phantom that is used to evaluate the PF approach. Section 4 then discusses the results and how this additional step further reduces errors, followed by concluding remarks and notes on further work in Section 5.

\section{METHODOLOGY}

\subsection{Particle Filter Implementation}

The PF uses a Bayesian view of the motion estimation problem and represents it as a first-order hidden Markov model, with the state $\mathbf{X}_{\mathrm{k}}$ being a hidden random variable and only correlated to its value at the previous time step $k-1$. At each time step, the hidden state is also correlated to observations $\mathbf{Z}_{\mathrm{k}}$ which are independent of each other. This HMM structure is shown in Figure 1 and this generic nonlinear dynamic system has the following state space representation:

$$
\begin{gathered}
\mathbf{X}_{k}=\mathbf{a}\left(\mathbf{X}_{k-1}, \mathbf{v}_{k-1}\right) \leftrightarrow \overbrace{f\left(\mathbf{X}_{k} \mid \mathbf{X}_{k-1}\right)}^{\text {Transition probability density }} \\
\mathbf{Z}_{k}=\mathbf{b}\left(\mathbf{X}_{k}, \mathbf{w}_{k}\right) \leftrightarrow \overbrace{g\left(\mathbf{Z}_{k} \mid \mathbf{X}_{k}\right)}^{\text {Measurement probability density }},
\end{gathered}
$$

where the models $\mathbf{a}$ and $\mathbf{b}$ have respective stochastic components $\mathbf{v}_{\mathrm{k}-1}$ and $\mathbf{w}_{\mathrm{k}}$. The stochastic components represent the uncertainties in the models and they give rise to the respective probability densities $f$ and $g$.

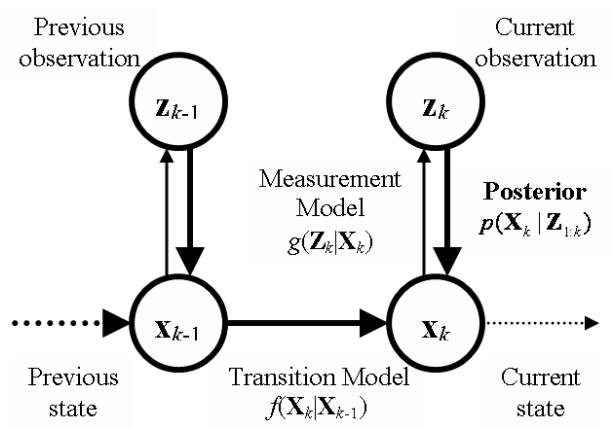

Figure 1. Diagram of the system in state-space form illustrating the structure as a first order hidden Markov model. The posterior of the current state is represented by the bolded arrows and circles showing the information available at time $\mathrm{k}$.

At any time $k$, an estimate of the state can be inferred from the posterior probability density $p\left(\mathbf{X}_{k} \mid \mathbf{Z}_{1: k}\right)$ which is conditional on the set of all observations up until the present time $k, \mathbf{Z}_{1: k} \equiv\left\{\mathbf{Z}_{1}, \ldots, \mathbf{Z}_{k}\right\}$. Some moment of the posterior can be taken as the actual state estimate, such as its expected value $\mathrm{E}\left[\mathbf{X}_{\mathrm{k}}\right]$. In a PF, the posterior is estimated as

$$
p\left(\mathbf{X}_{k} \mid \mathbf{Z}_{1: k}\right) \approx \sum_{i=1}^{N} w_{k}^{i} \delta\left(\mathbf{X}_{k}-\mathbf{X}_{k}^{i}\right)
$$


where the posterior is sampled along the space of $\mathbf{X}_{\mathrm{k}}$ (i.e. possible states) by a weighted impulse train represented by point masses (i.e. particles) $\mathbf{X}_{k}^{i}$. The particles are Monte Carlo samples of the state space and the probability of the posterior at the locations of the particles are given by their respective weights, $w_{k}^{i}$. As in Abd. Rahni et al., 2010 $0^{4}$, the $\mathrm{PF}$ is implemented as a sampling importance resampling (SIR) filter. The three application specific adaptation methods that have been previously implemented are also included in the current approach. These adaptation methods are:

1) Dimensionality Reduction using principal component analysis (PCA),

2) Incorporation of estimated respiratory parameters, and

3) Planned sampling of particles.

The purpose of these adaptation methods is to improve particle diversity and make the sampling of the state space more efficient. They have been outlined in Abd. Rahni et al., $2010^{4}$.

\subsection{Transition and Measurement Models}

Similar to the previous implementation in Abd. Rahni et al., 2010 , the state transition model a is chosen to be a secondorder autoregressive model, $\mathrm{AR}(2)$, as it is postulated that this would still reasonably represent the pseudo-oscillatory nature of respiratory motion. As the AR(2) model tracks organ configuration in another variable $\mathbf{x}_{\mathrm{k}}$, the state $\mathbf{X}_{\mathrm{k}}$ can be made to consist of two time points, $\mathbf{x}_{\mathrm{k}}$ and $\mathbf{x}_{\mathrm{k}-1}$. Hence in $\mathbf{X}_{\mathrm{k}}$, the transition model $\mathbf{a}$ is still a first-order Markov model and its corresponding probability density $f$ will be Gaussian. The parameters for a are found from stepwise least squares (LS) estimation on a training dataset.

Likewise, the measurement density $g$ is also chosen to be Gaussian. Its generative form $\mathbf{b}$, is a linear map of the state with a stochastic component:

$$
\mathbf{Z}_{k}=\beta \mathbf{X}_{k}+\boldsymbol{\beta}_{0}+M \mathbf{w}_{k}
$$

where $\mathbf{X}_{\mathrm{k}}$ consists of organ configurations from two time points as defined for the transition model, $\mathbf{w}_{\mathrm{k}}$ being a random vector consisting of independent standard normal variables giving $\mathbf{Z}_{\mathrm{k}}$ a covariance of $\Sigma_{\mathbf{Z}}=M M^{\mathrm{T}}$. This covariance accounts for the estimated inaccuracy of the map constants $\left(\beta\right.$ and $\left.\boldsymbol{\beta}_{0}\right)$ and observation noise. As in Abd. Rahni et al., $2010^{4}$, the observation noise is assumed to be isotropic with an root mean square error (RMSE) characteristic of the Polaris $^{\dagger}$ 3D optical stereo tracking system as it has been used before for motion correction in NM imaging such as for brain imaging ${ }^{8}$. The parameters for (4) are also found from LS estimation on a training dataset.
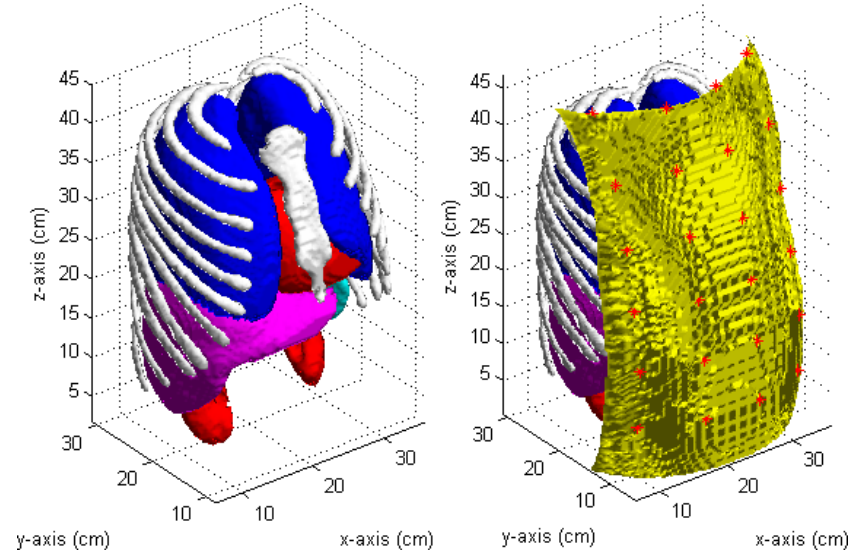

Figure 2. Left: Surface render of the chosen organs. Right: Surface render including an anterior portion of the external surface of the torso. The points chosen as the observable are marked in red $(*)$.

\footnotetext{
${ }^{\dagger}$ Northern Digital Inc.
} 


\subsection{Tracked State}

The organ configurations that are tracked, $\mathbf{x}_{\mathrm{k}}$, are affine transformation parameters for each organ $o$ at time $k$ for registration back to a baseline configuration $\theta$, selected from the training dataset. The organs chosen are the heart, liver, spleen, kidneys, lungs and ribcage. A surface render of these organs obtained from the XCAT phantom are shown in Figure 2. The transformation parameters are found from iterative closest points registration as explained in Jones et al., $2009^{5}$. On the other hand, the observations $\mathbf{Z}_{\mathrm{k}}$, are the 3D coordinates of a grid of point on the anterior surface of the external surface of the torso obtained from the XCAT phantom. The coronal projections of the points are set to be $7.8 \mathrm{~cm}$ apart from each other. Figure 2 also shows this external surface and the points chosen as the observables.

\subsection{Iterative Model Update}

Iterative model update constitute an additional application specific adaptation method that mainly seek to improve the distribution of the stochastic components of the transition and measurement models which are represented respectively by $\mathbf{v}_{\mathrm{k}-1}$ and $\mathbf{w}_{\mathrm{k}}$ in (1) and (2). The models are updated by incorporating observations and initial estimates of all test datasets into a subsequent training stage to re-estimate the transition and measurement models. This is possible as the motion estimation framework used is an offline approach. Figure 3 illustrates the model update step. During subsequent iterations of the PF approach, the PF is run without adaptation methods (2) and (3) as the updated models now have probability distributions which better reflect the variations across the test datasets. The effect this has on the adaptability of the transition and measurement models will be discussed in Section 4 along with its effect on motion estimation.

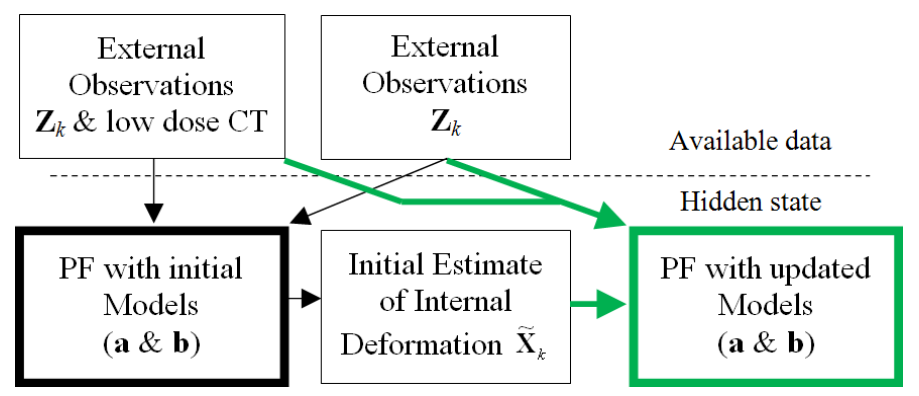

Figure 3. Diagram illustrating the model update procedure indicated by the green bolded arrows and box. The sequence of estimating $\widetilde{\mathbf{X}}_{k}$, then updating the models ( $\left.\mathbf{a} \& \mathbf{b}\right)$ can be iterated multiple times.

\section{EVALUATION}

The data used for training and evaluation is generated from XCAT to represent a male of $192 \mathrm{~cm}$ weighing $95.05 \mathrm{~kg}$. The voxel size is $(3.25 \mathrm{~cm})^{3}$ and the frame rate is $2 \mathrm{~s}^{-1}$. Evaluation is performed by comparing the position of organ voxels estimated by the PF with ground-truth positions provided by XCAT. The observable is also obtained from XCAT, but with simulated camera noise with the same RMSE as used in (4) and has a frame rate of $6 \mathrm{~s}^{-1}$.

Training data consists of a single respiratory cycle with diaphragm superior-inferior (SI) motion of $2 \mathrm{~cm}$ and ribcage anterior-posterior (AP) motion of $1.2 \mathrm{~cm}$. Consequently the observable has similar AP motion. The training cycle period is $5 \mathrm{~s}$. These parameter values for the training cycle represent average values for normal respiration. Respiration is assumed to start at the end of expiration, constituting the rest phase in XCAT. This rest phase is thus designated as the baseline configuration, $\theta$.

Test data consists of 7 datasets that have SI and AP motion that vary between 0.5 to 2 times of that of the training cycle. The test data also have cycle periods of either 3 or $5 \mathrm{~s}$. The parameters for the training cycle is also used as a self-test, albeit with a different realization of camera noise. This is designated as Test 0 in Table 1, along with the other 6 datasets. Section 4 will discuss the results in motion estimation using these datasets and the effect the iterative PF approach has on the estimation accuracy. 
Table 1. Details of dataset parameters used for evaluating the iterative PF approach.

\begin{tabular}{|c|c|c|c|}
\hline \multirow{2}{*}{ Dataset } & \multicolumn{2}{|c|}{ Motion amplitude (cm) } & \multirow{2}{*}{ Cycle Period (s) } \\
\cline { 2 - 4 } & Diaphragm (SI) & Chest (AP) & 5 \\
\hline Test 0 & 2 & 1.2 & 5 \\
\hline Test 1 & 1 & 0.6 & 5 \\
\hline Test 2 & 3 & 1.8 & 5 \\
\hline Test 3 & 4 & 0.6 & 3 \\
\hline Test 4 & 1 & 1.2 & 3 \\
\hline Test 5 & 2 & 1.8 & 3 \\
\hline Test 6 & 3 & & \\
\hline
\end{tabular}

\section{RESULTS AND DISCUSSION}

\subsection{Effect of Model update in adjusting the Particle Filter probability densities}

In the previous implementation in Abd. Rahni et al., $2010^{4}$, the PF approach was more dependent on the three adaptation methods that have been mentioned in Section 2.1. Moreover, in using adaptation method 1, the noise variance component of the first principal component (PC) of the projected state in the transition model is also increased by the amount given by an AR(2) approximation using only the first PC. This is based on results in Wells et al., 2009². Thus the covariance of the noise component of the transition model $\left(\Sigma_{\mathrm{v}}\right)$, is shown in Figure 4(a), which can be seen to be dominated by the additional added amount from the 1-D AR(2) approximation. Also shown in Figure 4(b) is the covariance of the measurement density $\left(\Sigma_{\mathbf{Z}}\right)$, which is similarly dominated by assumptions on the amount of measurement noise present in the stereo camera system that is used. These probability densities are thus limited by the assumptions made and the data used to construct the models used in the PF. This is why additionally, adaptation methods 2 and 3 are used to improve particle diversity and make sampling of the state space more efficient.

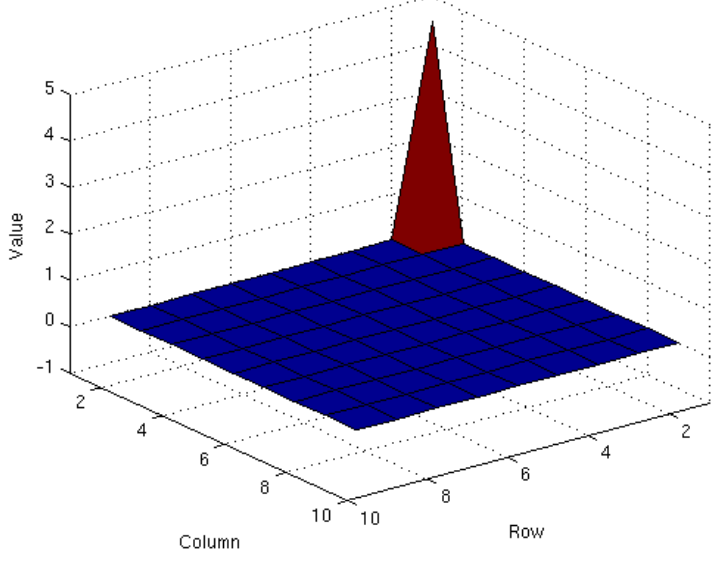

a) Covariance matrix of the noise component of the transition model, $\left(\Sigma_{\mathbf{v}}\right)$.

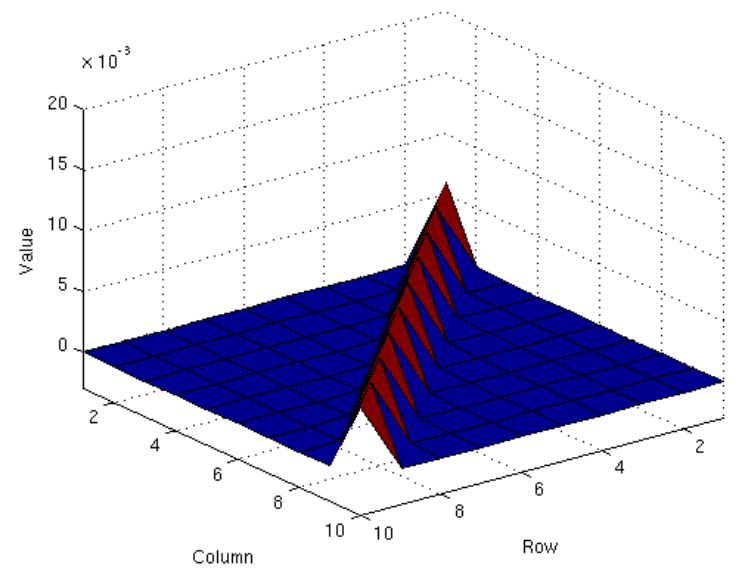

b) Covariance matrix of the measurement density, $\left(\Sigma_{\mathbf{Z}}\right)$.

Figure 4. Surface plots of the covariance matrices of the initial models in the PF.

When the models are updated by incorporating observations and initial estimates of the test datasets, the model probability densities used in the PF are modified so that they encompass all variation that have been seen. In this case adaptation method 1 is only used to project the state and observation into their respective PCA spaces. There is no 
further need to adjust the covariance of the models as they now reflect the test data better. The covariance matrices related to the PF models are now as shown in Figure 5. With this, there is no further need to use the additional adaptation methods 2 and 3 .

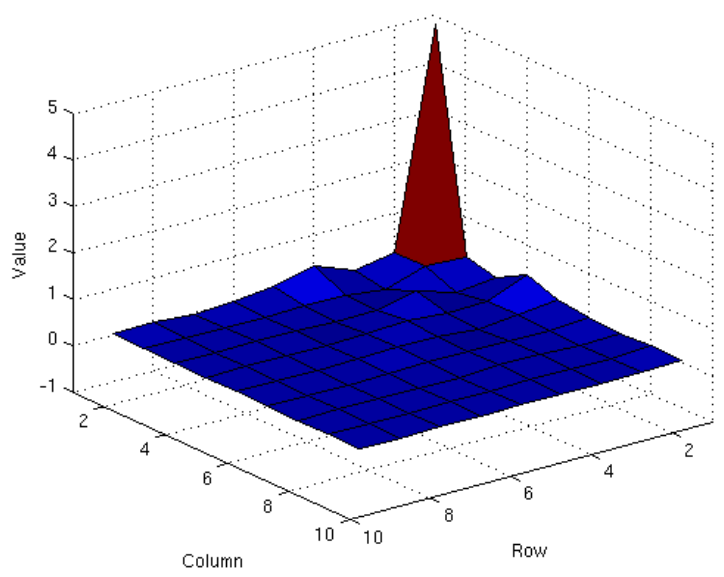

a) Covariance matrix of the noise component of the transition model, $\left(\Sigma_{\mathbf{v}}\right)$.

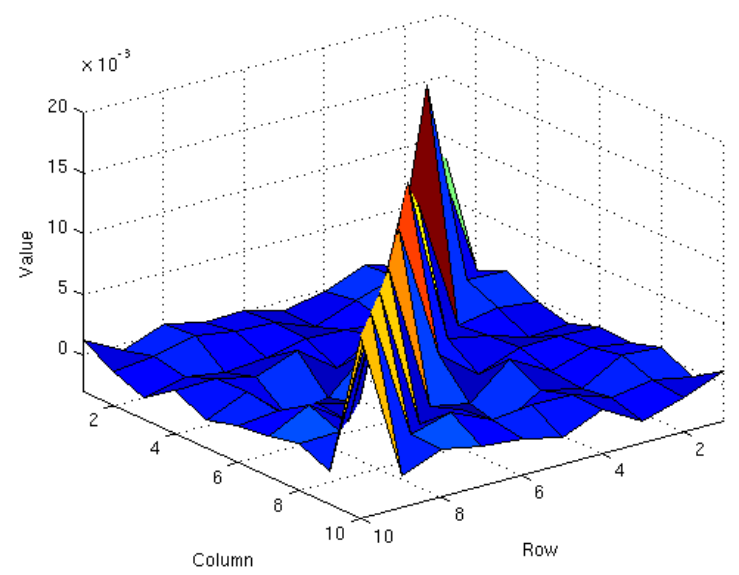

b) Covariance matrix of the measurement density, $\left(\Sigma_{\mathbf{Z}}\right)$.

Figure 5. Surface plots of the covariance matrices of the updated models in the PF.

\subsection{Effect of Iterating Model Update on Motion Estimation}

The accuracy of motion estimation is quantified by the error in the position of voxel centres that are registered back to the reference frame, $\theta$. These Euclidean distance errors are known in the simulated data as XCAT enables the displacement of any particular point within the body to be identified at any time point. Figure 6 shows the Euclidean distance errors averaged over all voxels on a per-organ basis over all test data for the PF approach after four iterations of the model update method. The errors are contrasted with the intrinsic errors from ICP registration and shows that the PF results in the about the same, if not less errors compared to those produced from ICP registration. The errors are especially low for organs besides the ribcage and lungs, about half of that produced from ICP registration. This is postulated by the face that affine transformation describes the motion of those organs better, thus the models can be optimised much further with respect to them. The variance over all test data indicated by the error bars are also only slightly larger than those from ICP registration. Note that ICP registration assumes knowledge of organ deformation, implying that the $\mathrm{PF}$ is able to accurately estimate the transformation parameters that describes that deformation.

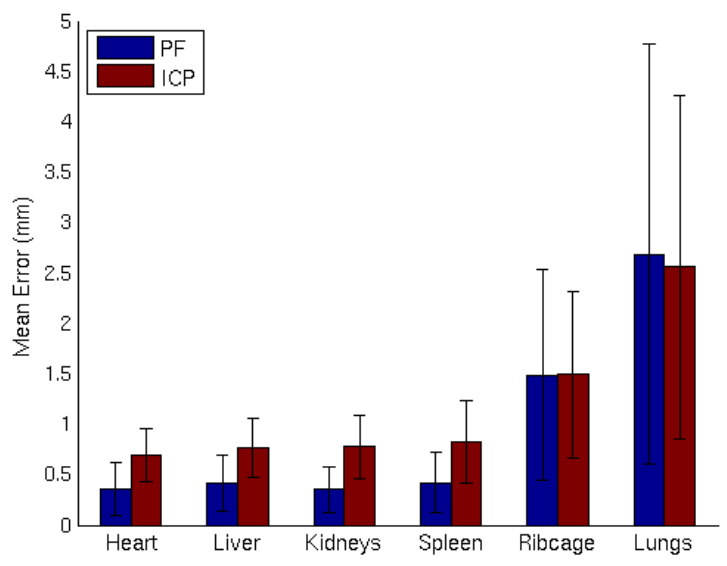

Figure 6. Mean errors for the PF after four iterations of the model update method contrasted with mean errors for ICP registration. 
Following on, Figure 7 plots the mean errors of each organ with each additional iteration of the model update step as a percentage of their mean errors without model update. As with the discussion for Figure 6, the improvement is more evident with organs besides the ribcage and lungs. After four iterations of the model update method, the error for those organs is reduced to almost half of that without model update. However the gain in error reduction with each additional iteration number also drops rapidly. Thus in improving the accuracy of motion estimation, the model update step can be limited to the first few iterations. The results also shows that even though simplistic models are used in this basic implementation of the PF, the state can still be estimated accurately if the models can be made to reflect the variation in the hidden state that is to be estimated. Additionally, it has to be noted that besides the simulated camera noise, conditions in the XCAT phantom are perfect with regards to having pre-segmented organs and the limited respiratory variations that can be made (cycle amplitude and period). It is postulated that the model update step may be needed more with real data when there can be more respiratory variations between the training phase during the short period involving low dose CT scans and the estimation phase during the longer NM scan.

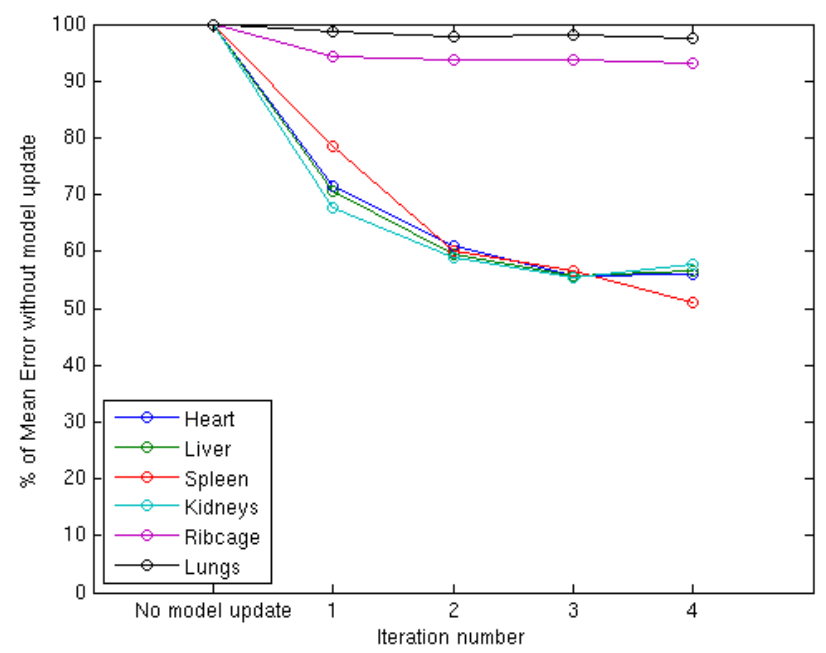

Figure 7. Plot of mean error for each organ as a percentage of that without model update versus model update iteration number.

\section{CONCLUSION}

The results in Figure 6 show that the accuracy of the PF is on average better than the voxel resolution and crucially for most organs less than half a voxel. The major source of error seems to be attributable to the assumption of affine transformation for respiratory motion, as shown by the intrinsic errors from ICP registration. The model update method appears to further decrease the mean error, especially for the heart, kidneys, liver and spleen. However, this may actually be due to simplifications within the XCAT phantom. The mean errors for the lungs and ribcage are decreased much less by the model update method than that of the other organs. Subsequent iterations of the model update method results in smaller improvement upon an additional iteration. This suggests that the iteration number can be limited to a small number.

Due to the limitations of the current simulation, further analysis is needed especially those using more realistic data. Future improvements are thus necessary as upcoming evaluations will use XCAT $2.0^{9}$ which should have more realistic respiratory motion simulated. Increased variation in test datasets using XCAT 2.0 is planned as is the incorporation of observables based on volunteer data. This can be contrasted with test derived from real data for a quantitative assessment that is nearer to clinical conditions. Further extension of the PF implementation such as those in Ristic, et al, $2004^{10}$ will be evaluated for this approach to cope with more realistic and complicated applications. 


\section{ACKNOWLEDGEMENT}

Ashrani Aizzuddin Abd. Rahni is funded by the Malaysian Ministry of Higher Education and University Kebangsaan Malaysia.

\section{REFERENCES}

[1] Lodge, M. A., Braess, H., Mahmoud, F., Suh, J., Englar, N., Geyser-Stoops, S., Jenkins, J., Bacharach, S. L., and Dilsizian, V., "Developments in Nuclear Cardiology, Transition from Single Photon Emission Computed Tomography to Positron Emission Tomography/Computed Tomography," J. Invasive Cardiol. 17(9), 491-496 (2005).

[2] Wells, K., Goswami, B., Abd Rahni, A. A., Jones, J., Alnowami, M., Lewis, E. B. and Guy, M., "A Flexible Approach to Motion Correction in Nuclear Medicine," in Conf. Rec. 2009 IEEE Nuclear Science Symposium/Medical Imaging Conference (NSS/MIC), 2534-2539 (2009)

[3] Segars, W. P., Mori, S., Chen, G.T.Y., and Tsui, B.M.W., "Modeling respiratory motion variations in the 4D NCAT phantom," in Conf. Rec. 2007 IEEE Nuclear Science Symposium/Medical Imaging Conference (NSS/MIC), 2677-2679 (2007).

[4] Abd. Rahni, A. A., E. Lewis, E., Guy, M. J., Goswami, B. and Wells, K., "Performance Evaluation of a Particle Filter Framework for Respiratory Motion Estimation in Nuclear Medicine Imaging," in Conf. Rec. 2010 IEEE Nuclear Science Symposium/Medical Imaging Conference (NSS/MIC), - (2010).

[5] Jones, J., Lewis, E., Guy, M. and Wells, K., "A virtual dissection based registration to model patient-specific respiratory motion," in Conf. Rec. 2009 IEEE Nuclear Science Symposium/Medical Imaging Conference (NSS/MIC), 3571-3576 (2009).

[6] Alnowami, M., Lewis, E., Guy, M. and Wells, K., "Marker-less Tracking for Respiratory Motion Correction in Nuclear Medicine Imaging," in Conf. Rec. 2010 IEEE Nuclear Science Symposium/Medical Imaging Conference (NSS/MIC), - (2010).

[7] Segars, W. P., [Development of a new dynamic NURBS-based cardiac-torso (NCAT) phantom], PhD thesis, The University of North Carolina, 118 (2001).

[8] Rahmim, A., Rousset, O., and Zaidi, H., "Strategies for motion tracking and correction in PET," PET Clin. 2(2), 251-266 (2007).

[9] Segars, W. P. and Tsui, B. M. W., "MCAT to XCAT: The Evolution of 4-D Computerized Phantoms for Imaging Research,” Proc. IEEE 97(12), 1954-1968 (2009).

[10] Ristic, B., Arulampalam, S. and Gordon, N., editors, [Beyond the Kalman Filter: Particle Filters for Tracking Applications], Artech House, - (2004). 